\title{
Relationship between age at onset and clinical characteristics of Behçet's disease
}

\author{
Mohammed El Amine Ghembaza ${ }^{1,2} \bowtie$, Ali Lounici $i^{1,2}$
}

\begin{abstract}
Introduction: This study compares the clinical characteristics and treatment modalities of Behçet's disease patients by age of onset at the Dr. Tidjani Damerdji University Hospital Center in Tlemcen, Algeria.

Methods: Data from patients treated for Behçet's disease between January 1990 and December 2016 were analyzed retrospectively. All patients that fulfilled the diagnostic criteria laid down by the International Study Group for Behçet's Disease were included in the study. Patients with early-onset disease ( $\leq 30$ years) were compared to those with late-onset disease ( $>30$ years).

Results: Among the 77 patients whose data were collected for the study, $80.5 \%$ were male and the mean age at onset was $27.2 \pm 7$ years. Approximately $60 \%$ of the patients had early-onset disease, and those patients exhibited more frequent intermediate uveitis ( $52.9 \%$ vs. $22.7 \%, p=0.03)$, posterior uveitis ( $54.3 \%$ vs. $23.8 \%, p=0.03)$, and panuveitis $(45.7 \%$ vs. $14.3 \%, p=0.01)$ compared to those with late-onset disease. Azathioprine ( $57.5 \%$ vs. $26.9 \%, p=0.01)$ and biological therapy $(15 \%$ vs. $0 \%, p=0.03)$ were used more frequently in the early-onset group.
\end{abstract}

Conclusions: Early-onset Behçet's disease was associated with more frequent and more severe ocular features. Furthermore, azathioprine and biological therapy were more often used in that group of patients.

Keywords: Behçet’s disease, vasculitis, early-onset, late-onset, epidemiology, Algeria

Received: 26 November 2017 | Returned for modification: 6 July 2018 | Accepted: 26 October 2018

\section{Introduction}

Behçet's disease (BD) is a multisystem relapsing vasculitis of unknown etiology. The hallmark signs of the disease are recurrent oral and genital ulcers. BD can also involve a broad array of systems-including ocular, cardiovascular, neurological, and gastrointestinal-throughout the disease course (1). Genetic background and environmental factors can affect its prevalence and clinical presentation (2). BD is most often found in the geographical region defined by the former Silk Road, which extends from the Mediterranean countries to the Middle East and Far East (2). The highest prevalence rates have been reported in Turkey (up to 421 per 100,000), and lower prevalence rates are seen in northern European countries and the United States $(3,4)$. It has long been accepted that the course of BD is more severe in younger patients (5). However, information is lacking about the relationship in Algeria between age at onset and the clinical characteristics of BD. The study described here compared the clinical characteristics and treatment modalities of BD by age at onset at the Dr. Tidjani Damerdji University Hospital Center in Tlemcen, Algeria.

\section{Methods}

This is a retrospective study of patients with BD that were admitted to the Internal Medicine Department at the Dr. Tidjani Damerdji University Hospital Center over a 16-year period from January 1990 to December 2016. Only patients that fulfilled the diagnostic criteria laid down by the International Study Group (ISG) for BD were included in the study (6). Data describing the clinical characteristics of the first 61 patients, collected between January 1990 and January 2015, have already been published (7).
The following data were collected on each patient: demographic variables, age at onset, age at diagnosis, organ involvement, management protocols, and HLA-B51 test results. An assessment of dermatological involvement was based on patient self-reporting and/or a clinical evaluation. A pathergy test was performed on the flexor forearm by inserting a 20-gauge intradermal sterile needle obliquely and then injecting $0.1 \mathrm{ml}$ of normal saline solution. The pathergy reaction was read at 48 hours, and the formation of a papule or pustule was considered a positive result. A diagnosis of ocular manifestations was made by an ophthalmologist, and an assessment of rheumatologic involvement was based on the patient's complaint and/or a physical examination. A diagnosis of cardiovascular manifestations was based on clinical indications, echocardiography, Doppler ultrasonography, and/ or angiography by means of computed tomography (CT) or magnetic resonance imaging (MRI). Neurological involvement was diagnosed based on a clinical assessment, cerebrospinal fluid analysis, and neuroimaging, including CT and/or MRI. Finally, symptomatic patients with gastrointestinal involvement confirmed by endoscopy and biopsy were diagnosed with entero-BD.

Age at onset was defined as the patient's age when BD first manifested. Age at diagnosis was defined as the patient's age when a healthcare professional informed the patient about the diagnosis of BD. Because BD tends to manifest in the third decade of life, patients were divided into two groups according to age at onset: patients with disease onset $\leq 30$ years were assigned to the early-onset group, and patients with disease onset $>30$ years were assigned to the lateonset group (8). To test for differences in proportions between the two groups, a chi-squared test was used. A $p$ value of 0.05 was considered statistically significant. Statistical analysis was performed with the Stastistical Package for Social Sciences (SPSS) version 17.0. 


\section{Results}

Among the 77 consecutive BD cases reviewed during the study period, 62 patients $(80.5 \%)$ were male, for a male-to-female ratio of 4:1. The mean \pm SD age at onset was $27.2 \pm 7$ years (8-43 years). The mean \pm SD age at diagnosis was $31.5 \pm 8.4$ years ( $13-56$ years), and the mean \pm SD time from onset to diagnosis was $4.19 \pm 6.2$ years (o-26 years). Table 1 shows the main characteristics of the patients. Forty out of 77 patients (59.7\%) had early-onset disease, and patients in that group exhibited significantly more frequent intermediate uveitis (52.9\% vs. $22.7 \%, p=0.03$ ), posterior uveitis (54.3\% vs. $23.8 \%, p=0.03$ ), and panuveitis ( $45.7 \%$ vs. $14.3 \%, p=$ o.01) compared to those in the late-onset group. The proportion of dermatological, joint, vascular, neurological, and gastrointestinal features was greater in the late-onset group, without reaching any statistical significance. Azathioprine and biological therapy were used more frequently in the early-onset group than in the lateonset group (57.5\% vs. $26.9 \%, p=0.01$ and $15 \%$ vs. $0 \%, p=0.03$, respectively). However, there were no statistically significant differences between the two groups in terms of the use of other drug treatments. Table 2 summarizes the main differences between the two groups.

\section{Discussion}

The aim of this study was to compare the clinical characteristics and treatment modalities of BD by age at onset at the Dr. Tidjani Damerdji University Hospital Center in Tlemcen, Algeria. The results show that patients with early-onset disease tended to have more frequent and severe uveitis and to require more treatment with azathioprine and biological therapies than did patients with late-onset disease. In this cohort, almost $60 \%$ of the patients had early-onset BD. It has long been known that BD patients with early onset show more major organ involvement and more severe disease activity (9). These data show that early-onset BD might also be an associated risk factor for more frequent and severe ocular manifestations. This finding is supported by the work reported by Citirik et al. (10). Furthermore, in the study conducted by Yazici et al. (9), patients with early-onset disease-of both sexes-experienced more ocular involvement than did patients with lateonset disease. However, the frequencies of mucocutaneous, joint, vascular, and neurological manifestations were not influenced by age at onset in the study by Yazici et al., matching the results of this study. It must be kept in mind that, in the afore mentioned study, patients 24 years old or younger at onset were assigned to the early-onset group (9). Furthermore, Talarico et al. (11) showed that $95 \%$ of patients that developed major organ involvement during follow-up had experienced disease onset before they were 25 years old with major organ involvement defined as eye disease, vascular involvement, and neurological involvement; this is only partially in agreement with the findings reported here.

Our results stand in contrast to the study by Alli et al., which showed no significant association between eye involvement and age at onset in Ankara, Turkey (12) and to the study by Whallett et al. that reported the same findings in the United Kingdom (13). Moreover, Sunger et al. failed to detect any significant difference between patients with and without ocular manifestations by age at onset; however, it should be taken into account that only patients that had their first symptoms after age 40 were included in the study (14). In the study by Karincaoglu et al., patients with juvenile onset disease showed more neurological and gastrointes-
Table 1 | Demographic and clinical characteristics of 77 patients with Behçet's disease.

\begin{tabular}{lcc}
\hline Patient characteristic & $n$ & $\%$ \\
\hline Age at onset (years), mean \pm standard deviation & $27.2 \pm 7$ & \\
Age at diagnosis (years), mean \pm Standard deviation & $31.5 \pm 8.4$ & \\
Sex & & \\
$\quad$ Male & 62 & 80.5 \\
$\quad$ Female & 15 & 19.5 \\
Dermatological features & 77 & 100.0 \\
$\quad$ Oral ulcers & 49 & 72.1 \\
$\quad$ Genital ulcers & 40 & 61.5 \\
Pseudofolliculitis & 9 & 14.3 \\
Erythema nodosum & 3 & 3.9 \\
Cutaneous ulcerations & 11 & 18.3 \\
Pathergy test positivity & 51 & 66.2 \\
Ocular features & 40 & 54.8 \\
Joint features & 20 & 26.0 \\
Vascular features & 17 & 22.4 \\
Neurological features & 2 & 2.7 \\
Gastrointestinal features & 3 & 4.1 \\
HLA-B51+ & & \\
Treatment protocol & 77 & 100.0 \\
$\quad$ Colchicine & 58 & 76.3 \\
Corticosteroids & 33 & 43.4 \\
Azathioprine & 10 & 13.2 \\
Cyclophosphamide & 7 & 9.0 \\
Biological therapy & 17 & 22.1 \\
Anticoagulants & & \\
\hline
\end{tabular}

Table 2 | Comparison between patients with early- versus late-onset Behçet's disease.

\begin{tabular}{|c|c|c|c|}
\hline Patient characteristic & $\begin{array}{c}\text { Early-onset group } \\
(\%)\end{array}$ & $\begin{array}{c}\text { Late-onset group } \\
(\%)\end{array}$ & $p$ value \\
\hline \multicolumn{4}{|l|}{ Sex } \\
\hline Male & 77.5 & 88.9 & 0.23 \\
\hline Female & 22.5 & 11.1 & \\
\hline \multicolumn{4}{|l|}{ Dermatological features } \\
\hline Oral ulcers & 100.0 & 100.0 & 1.00 \\
\hline Genital ulcers & 66.7 & 73.9 & 0.55 \\
\hline Pseudofolliculitis & 55.3 & 61.9 & 0.62 \\
\hline Erythema nodosum & 11.1 & 14.3 & 0.48 \\
\hline Cutaneous ulcerations & 2.5 & 7.4 & 0.34 \\
\hline Pathergy test & 14.7 & 28.6 & 0.20 \\
\hline Ocular features & 72.5 & 55.6 & 0.15 \\
\hline Anterior uveitis & 51.4 & 27.3 & 0.05 \\
\hline Intermediate uveitis & 52.9 & 22.7 & 0.03 \\
\hline Posterior uveitis & 54.3 & 23.8 & 0.03 \\
\hline Panuveitis & 45.7 & 14.3 & 0.01 \\
\hline Retinal vasculitis & 50.0 & 39.1 & 0.41 \\
\hline Joint features & 52.6 & 57.7 & 0.69 \\
\hline Vascular features & 20.0 & 33.3 & 0.21 \\
\hline Neurological features & 17.5 & 26.9 & 0.27 \\
\hline Gastrointestinal features & 2.6 & 3.8 & 0.78 \\
\hline HLA-B51+ & 5.3 & 3.7 & 0.92 \\
\hline \multicolumn{4}{|l|}{ Treatment protocol } \\
\hline Colchicine & 100.0 & 100.0 & 1.00 \\
\hline Corticosteroids & 77.5 & 73.1 & 0.68 \\
\hline Azathioprine & 57.5 & 26.9 & 0.01 \\
\hline Cyclophosphamide & 12.5 & 11.5 & 0.90 \\
\hline Biological therapy & 15.0 & 0 & 0.03 \\
\hline Anticoagulants & 15.0 & 29.6 & 0.14 \\
\hline
\end{tabular}

tinal involvement than did patients with adult onset disease (patients were characterized as adult onset BD if they had had their first symptoms after age 16); conversely, there was no difference between the two groups in terms of ocular manifestations (15).

Aside from age at onset, sex is one of the major prognostic factors of BD because men experience more major organ involvement, especially ocular and vascular manifestations (16). This study failed to show a statistical difference between the early- and late-onset groups in terms of sex. This is in line with the results 
reported by Hamzaoui et al. (17).

In agreement with earlier reports, the data from this study did not show any major differences between the early-onset and lateonset groups with regard to pathergy positivity and HLA-B51 status $(17,18)$, whereas a previous report uncovered a strong association between ocular manifestations and HLA-B51 positivity (19).

The difference between the two groups in terms of treatment regimens could be explained by the fact that the large majority of patients with severe ocular manifestations were treated with azathioprine and systemic corticosteroids, whereas those with other manifestations mostly were not. Moreover, when this combination therapy failed to achieve ocular remission, a biological therapy was added. In point of fact, according to the European League Against Rheumatism (EULAR) guidelines, azathioprine should be used as a first-line therapy, in combination with corticosteroids, in patients with posterior segment involvement. Infliximab is therefore reserved for patients with a sight-threatening eye disease, defined as retinal vasculitis and/or a severe decrease in visual acuity (20).

The definition of early-onset disease differs among studies, which may partially explain the conflicting results reported by the authors. In some studies, early-onset disease included only those whose disease was fully manifested and diagnosed in childhood, whereas other studies, like ours, included patients in the earlyonset group that exhibited their first symptoms in childhood but the full disease emerged later. Other factors, such as differences in geographical and ethnic origin and in study design, may also account for the conflicting results reported in the literature. Comparisons between studies would be misleading because different definitions of early-onset disease have been used. Indeed, there is no standard definition of early-onset $\mathrm{BD}$, and thus many ages have been set as cutoff points by different authors. However, because BD occurs most often during the third decade of life, it was reasonable to use 30 years as the cutoff point in this study.

Our study has several limitations. Because the cohort came from a hospital, the study tended to include more severe cases, which may explain the high prevalence of ocular and vascular involvement. Moreover, patients with noninfectious uveitis are often referred to the internal medicine department for further diagnostic assessment; this could explain, at least in part, the high proportion of ocular manifestations in both groups.

\section{Conclusion}

In this study, mucocutaneous lesions, ocular involvement, and articular involvement were the most common symptoms of BD. Furthermore, patients with early-onset disease tended to have more frequent and severe uveitis and required relatively more azathioprine and biological therapies than did patients with lateonset disease. Therefore, tight control over the treatments used is strongly recommended during the course of the disease in those patients.

\section{References}

1. Sakane T, Takeno M, Suzuki N, Inaba G. Behçet's disease. N Engl J Med. 1999; 341:1284-91.

2. Poon W, Verity DH, Larkin GL, Graham EM, Stanford MR. Behçet's disease in patients of West African and Afro-Caribbean origin. Br J Ophthal. 2003;87:876-8.

3. Azizlerli G, Köse AA, Sarıca R, Gül A, Tutkun IT, Kulaç M, et al. Prevalence of Behçet's disease in Istanbul, Turkey. Int Journal Dermatol. 2003;42:803-6.

4. Leonardo NM, McNeil J. Behçet's disease: is there geographical variation? A review far from the Silk Road. Int Journal Rheumatol. 2015;2015:945262.

5. Zeidan MJ, Saadoun D, Garrido M, Klatzmann D, Six A, Cacoub P. Behçet's disease physiopathology: a contemporary review. Auto Immun Highlights. 2016; $7: 4$.

6. International study group for Behcet's disease. Criteria for diagnosis of Behçet's disease. Lancet. 1990;335:1078-80.

7. Ghembaza M, Bouabdallah N, Lounici A. Behçet disease in western Algeria. Med Sante Trop. 2017;27:101-4.

8. Zouboulis CC. Epidemiology of Adamantiades-Behçet's disease. Ann Med Interne (Paris). 1999;150:488-98.

9. Yazici H, Tüzün Y, Pazarli H, Yurdakul S, Ozyazgan Y, Ozdoğan H, et al. Influence of age of onset and patient's sex on the prevalence and severity of manifestations of Behcet's syndrome. Ann Rheumatic Dis. 1984;43:783-9.

10. Citirik M, Berker N, Songur MS, Soykan E, Zilelioglu O. Ocular findings in childhood-onset Behçet disease. J AAPOS. 2009;13:391-5.

11. Talarico R, Cantarini L, d'Ascanio A, Figus M, Favati B, Baldini C, et al. Development of de novo major involvement during follow-up in Behçet's syndrome. Clin Rheumatol. 2016;35:247-50.

12. Tugal-Tutkun I, Onal S, Altan-Yaycioglu R, Altunbas HH, Urgancioglu M. Uveitis in Behçet disease: an analysis of 880 patients. Am Journal Ophthalmol. 2004;138: 373-80.
13. Whallett AJ, Thurairajan G, Hamburger J, Palmer RG, Murray PI. Behçet's syndrome: a multidisciplinary approach to clinical care. QJM. 1999;92:727-40.

14. Sungur G, Hazirolan D, Hekimoglu E, Kasim R, Duman S. Late-onset Behçet's disease: demographic, clinical, and ocular features. Graefes Arch Clin Exp Ophthalmol. 2010;248:1325-30.

15. Karincaoglu Y, Borlu M, Toker SC, Akman A, Onder M, Gunasti S, et al. Demographic and clinical properties of juvenile-onset Behçet's disease: a controlled multicenter study. J Am Acad Dermatol. 2008;58:579-84.

16. Bonitsis NG, Luong Nguyen LB, LaValley MP, Papoutsis N, Altenburg A, Kötter I, et al. Gender-specific differences in Adamantiades-Behçet's disease manifestations: an analysis of the German registry and meta-analysis of data from the literature. Rheumatology (Oxford). 2014:54:121-33.

17. Hamzaoui A, Jaziri F, Ben Salem T, Ben Ghorbel FSI, Lamloum M, Smiti Khanfir $M$, et al. Comparison of clinical features of Behcet disease according to age in a Tunisian cohort. Acta Med Iran. 2014;52:748-51.

18. Sungur GK, Hazirolan D, Yalvac I, Ozer PA, Yuksel D, Vural ET, et al. Clinical and demographic evaluation of Behçet disease among different paediatric age groups. Br J Ophthalmol. 2009;93:83-7.

19. Paovic J, Paovic P, Sredovic V. Behcet's disease: systemic and ocular manifestations. Biomed Res Int. 2013;2013:247345.

20. Hatemi G, Silman A, Bang D, Bodaghi B, Chamberlain A, Gul A, et al. EULAR recommendations for the management of Behçet disease. Ann Rheum Dis. 2008; 67:1656-62. 